\title{
Characterization of Barley Entries for Spot Blotch Resistance
}

\author{
Amrinder Kaur ${ }^{1 *}$, Vineet K. Sharma ${ }^{1}$, Simarjit Kaur ${ }^{2}$, Jaspal Kaur ${ }^{2}$ and Chunni Lal ${ }^{3}$ \\ ${ }^{1}$ Department of Plant Pathology, ${ }^{2}$ Department of Plant Breeding and Genetics, Punjab \\ Agricultural University, Ludhiana-141004, Punjab, India \\ ${ }^{3}$ ICAR-Indian Institute of Wheat and Barley Research, Karnal-132001, Haryana, India \\ *Corresponding author
}

\section{A B S T R A C T}

\begin{tabular}{l}
\hline K e y w o r d s \\
$\begin{array}{l}\text { Barley, Spot blotch, } \\
\text { Bipolaris } \\
\text { sorokiniana, } \\
\text { screening }\end{array}$ \\
\hline Article Info \\
\hline $\begin{array}{l}\text { Accepted: } \\
\text { 04 September } 2020 \\
\text { Available Online: } \\
10 \text { October } 2020\end{array}$ \\
\hline
\end{tabular}

Barley (Hordeum vulgare L.) belonging to Gramineae family is an important cereal in the countries which have arid and semi-arid type. Among the different diseases occurring on barley, spot blotch or foliar blight caused by Bipolaris sorokiniana (Sacc.) Shoemaker is more damaging than other diseases, causing major reduction in quality and grain yield of barley crop. This disease can be managed by repeated fungicide applications, however, deployment of resistant varieties still remains on the top priority. Out of the 262 barley entries screened for two consecutive years under artificial epiphytotic conditions, five entries consisting of four germplasm lines viz., BL-1309, BL-1313, BL-1532, BL-1562 and one variety PL-891 were resistant towards the disease whereas thirty-three entries and two hundred and eighteen entries exhibited moderately resistant and moderately susceptible reaction respectively. Disease score of more than 78 was recorded in six entries namely, BL-1500, BL-1540, BL-1542, BL-1576, BL-1652 and PL-426 (susceptible check), exhibiting susceptible reaction towards disease. Thus, the barley entries which are resistant to spot blotch disease under artificial inoculated conditions during two years of testing under field conditions can be utilized as donars by breeders to incorporate spot blotch resistance in good yielding cultivars, which are prior found to be susceptible to the disease.

\section{Introduction}

Barley (Hordeum vulgare L.) is an important cereal of Gramineae family which is serving as the major portion of diet of the people and feed for animals. In the world, barley is cultivated on an area of nearly 50 million hectares with annual production of more than 140 million tons (Mt) (Tricase et al., 2018). It is a dominant crop in the countries which have arid and semi-arid type of climate, which thus favours development and yield of the crop. In the world, following wheat, maize and rice crop, barley occupies the fourth position as important cereal based crop belonging to graminaceous family (Taner et $a l ., 2004)$. In Punjab, barley covers an area of 7.7 thousand hac with production of 30,000 tonnes and average yield of 38.80 quintals/hectare (Anonymous 2019). Due to damaging effect of different diseases, pests and poor crop management practices, the 
average yield of barley in India is reported to be comparatively lower than several other countries. Among the different diseases occurring on barley, spot blotch or foliar blight caused by Bipolaris sorokiniana (Sacc.) Shoemaker (teleomorph: Cochliobolus sativus) is more damaging than other diseases, causing major reduction in grain quality and yield of this crop (Nutter et al., 1985; Arabi and Jawahar 2003). Yield losses of 25-45\% in barley crop have been reported in Kazakhstan and $41 \%$ in Russia due to this pathogen (Iftikhar et al., 2009). Although spot blotch is generally associated with warm conditions, but a survey of barley diseases conducted in higher altitudes of Trans Himalayan - Ladakh region of Indiaby Vaish et al., (2011) estimated yield losses of $6 \%$ to $53 \%$ due to $B$. sorokiniana causing spot blotch of barley.

The infection of this disease at seedling stage starts as small brownish black spots on leaf sheaths and which thus progresses from lower to upper plant parts during crop development (Kutcher et al., 1994). Higher level of resistance in different barley germplasm lines is difficult to achieve owing to the genetic changes in the pathogen population, influence of the environment on disease development and the quantitative nature of resistance (Wilcoxson et al., 1990). The spot blotch disease frequently hinders the commercial production of barley in the north-eastern states of India. Due to the changing agronomic practices and the quick replacement of local varieties with highyielding cultivars have thus led to the appearance of this disease in the northwestern regions of the country as well where it was earlier found to be insignificant (Bala and Kaur 2008). Foliar fungicide treatments can be used to control the spot blotch on barley, however, considering the environmental issues, deployment of resistant cultivars still remains on the top priority and thus offers the most economically and environmentally safe means of disease control (Singh et al., 2017). Thus, the aim of the present study was to evaluate different barley accessions/germplasm lines and released popular varieties for two successive years under artificial inoculated conditions so as to identify resistance sources against spot blotch disease which could serve as donors in breeding for resistance programmes.

\section{Materials and Methods}

The material under study consisted of two hundred and sixty-two entries including released varieties and advanced breeding lines procured from Department of Plant Breeding and Genetics, Punjab Agricultural University, Ludhiana and Indian Institute of Wheat and Barley Research (IIWBR), Karnal. In order to find new resistance sources among these barley entries against Bipolaris sorokiniana, field experiments were conducted for two successive cropping seasons i.e. 2017-18 and 2018-19.Each entry was sown as paired rows of one meter length with $20 \mathrm{~cm}$ distance between rows along with susceptible check i.e. PL 426 which was repeated after every 10 entries.

Mass inoculum of the pathogen was prepared in the laboratory by inoculating pure culture of the pathogen i.e. Bipolaris sorokiniana on autoclaved sorghum seeds in separate flask, which were then kept in incubator at $25 \pm 2^{\circ} \mathrm{C}$. After the full growth of the pathogen on the substrate, spores were harvested in distilled water which act as conidial suspension. All these barley entries were spray inoculated at maximum tillering stage with conidial suspension having spore concentration of $10^{6}$ conidia/ml during evening hours for ensuring successful infection (Chaurasia et al., 1999). After 12 days of inoculation, the characteristic brown coloured spots developed on leaves which further coalesce to form a large necrotic area thus producing the 
characteristic symptom of spot blotch disease. Disease assessment was done by recording the disease severity on leaves at three different crop growth stages viz. flowering, milk and hard dough stage by employing the standard double digit scale (00-99) given by Saari and Prescott (1975).

The left and right side of the double digit indicate the per cent disease severity score of blight on flag leaf (F) and flag-1 leaf (F-1) respectively. Since these two leaves remain green at milk stage and contribute most to the grain filling process, hence reduction of grain yield is directly related to disease severity in these two leaves (Singh et al., 2005). The area under disease progress curve (AUDPC) was also calculated for each entry from the disease score recorded at weekly intervals at different growth stages by the formula given by Roelfs et al., (1992) and all the entries were categorized based on their AUDPC values and terminal disease severity by using described scale ranging from highly resistant to susceptible (Table 1) (Van der Plank, 1968; Duveiller et al., 1998).

$$
\operatorname{AUDPC}=\sum_{\mathrm{i}=1}^{\mathrm{a}}\left[\left\{\left(\mathrm{Y}_{\mathrm{i}}+\mathrm{Y}_{(\mathrm{i}+1)}\right) / 2\right\} \times\left(\mathrm{t}(\mathrm{i}+1)-\mathrm{t}_{\mathrm{i}}\right)\right]
$$

Where,

$\mathrm{Yi}=$ disease score at time ti and,

$\mathrm{X}_{\mathrm{i}}$ and $\mathrm{X}_{\mathrm{i}+1}$ are disease severity on $\mathrm{i}$ and $\mathrm{i}+1$ date, respectively

$t_{i}$ is the no. of days between $i$ and $i+1$

$\mathrm{n}$ is the number of observations recorded.

\section{Results and Discussion}

The most important measure to counteract the attack of pathogen for longer durations is use of resistant cultivars. The results obtained from the evaluation of two hundred and sixtytwo barley entries evaluated for two consecutive years against $B$. sorokiniana under artificial inoculated conditions, revealed that all the entries showed almost similar reaction towards the disease during both the years of testing (Table 2). None of the cultivar or germplasm line exhibited highly resistant reaction.

Five entries consisting of four germplasm lines viz., BL-1309, BL-1313, BL-1532, BL1562 and one variety PL-891 were resistant towards the disease having disease score of less than 35, whereas disease score between 36 to 57 was observed in 33 entries, thus exhibiting moderately resistant reaction. Disease score of more than 69 was recorded in six entries namely, BL-1500, BL-1540, BL-1542, BL-1576, BL-1652 and PL-426 (susceptible check), exhibiting susceptible reaction towards disease, while in rest of the entries, disease score was recorded between 58-69, thus exhibiting moderately susceptible reaction (Table 3 ).

In context with the present findings, Verma et al., (2013) had also carried out multilocation evaluation of 5458 barley germplasm accessions for resistance to spot blotch under artificial inoculated conditions for four cropping seasons at four different locations and reported that out of these accessions, only 28 accessions were found to be resistant towards spot blotch disease. Similarly, eighty five barley germplasm accessions were screened under artificial inoculated conditions against leaf blight disease by Jain et al., (2014) and out of which 68 entries were found to be resistant while rest of the entries exhibited moderately resistant to susceptible reaction towards disease. Singh et al., (2018) also evaluated 62 wheat genotypes under natural epiphytotic conditions against Bipolaris sorokiniana, among which eight genotypes having disease severity between 34.26 to 35.0 per cent were observed as resistant. 
Table.1 Categorization of disease reaction based on severity score of spot blotch disease

\begin{tabular}{|l|l|c|}
\hline Sr. No. & Disease Reaction & Range of values (DD)* \\
\hline $\mathbf{1}$ & Immune & $00-01$ \\
\hline $\mathbf{2}$ & Resistant (R) & $12-24$ \\
\hline $\mathbf{3}$ & Moderately Resistant (MR) & $34-46$ \\
\hline $\mathbf{4}$ & Moderately Susceptible (MS) & $56-68$ \\
\hline $\mathbf{5}$ & Susceptible (S) & $78-89$ \\
\hline $\mathbf{6}$ & Highly Susceptible (HS) & 99 \\
\hline
\end{tabular}

*First and second value respectively, represents percent blighted area on the flag leaf and flag-1 leaves. Values $1,2,3,4,5,6,7,8$, and 9 , respectively correspond to $10,20,30,40,50,60,70,80$ and 90 percent blighted area

Table.2 Reaction of different barley entries against B. sorokiniana during two consecutive years of testing (2017-18 and 2018-19)

\begin{tabular}{|c|c|c|c|c|c|c|c|c|c|c|}
\hline \multirow[t]{2}{*}{$\begin{array}{l}\text { Germplasm lines/ } \\
\text { varieties }\end{array}$} & \multicolumn{3}{|c|}{ Foliar blight score (dd)* } & \multirow[t]{2}{*}{ AUDPC** } & \multirow[t]{2}{*}{ DR** } & \multicolumn{3}{|c|}{$\begin{array}{c}\text { Foliar blight score } \\
\text { (dd)* }\end{array}$} & \multirow[t]{2}{*}{ AUDPC } & \multirow[t]{2}{*}{ DR** } \\
\hline & $\mathbf{F}$ & D & H D & & & $\mathbf{F}$ & D & H D & & \\
\hline & \multicolumn{5}{|c|}{ 2017-18 } & \multicolumn{5}{|c|}{ 2018-19 } \\
\hline BL 1301 & 23 & 35 & 58 & 372.5 & MS & 22 & 34 & 58 & 365.0 & MS \\
\hline BL 1309 & 11 & 12 & 23 & 145.0 & $\mathrm{R}$ & 12 & 14 & 24 & 160.0 & $\mathrm{R}$ \\
\hline BL 1313 & 1 & 11 & 23 & 115.0 & $\mathrm{R}$ & 11 & 12 & 23 & 145.0 & $\mathrm{R}$ \\
\hline BL 1314 & 12 & 24 & 35 & 237.5 & MR & 12 & 24 & 35 & 237.5 & MR \\
\hline BL 1319 & 13 & 35 & 58 & 362.5 & MS & 13 & 37 & 58 & 365.0 & MS \\
\hline BL 1322 & 13 & 36 & 58 & 365.0 & MS & 12 & 25 & 58 & 297.5 & MS \\
\hline BL 1325 & 12 & 26 & 46 & 275.0 & MR & 12 & 26 & 47 & 277.5 & MR \\
\hline BL 1335 & 24 & 36 & 59 & 387.5 & MS & 24 & 35 & 58 & 380.0 & MS \\
\hline BL 1338 & 25 & 36 & 58 & 387.5 & MS & 25 & 36 & 58 & 387.5 & MS \\
\hline BL 1340 & 22 & 34 & 58 & 365.0 & MS & 22 & 35 & 58 & 367.5 & MS \\
\hline BL 1363 & 24 & 37 & 58 & 390.0 & MS & 24 & 37 & 58 & 390.0 & MS \\
\hline BL 1367 & 25 & 47 & 69 & 470.0 & MS & 25 & 46 & 68 & 462.5 & MS \\
\hline BL 1368 & 22 & 34 & 58 & 365.0 & MS & 22 & 35 & 58 & 367.5 & MS \\
\hline BL 1669 & 24 & 45 & 58 & 425.0 & MS & 24 & 36 & 58 & 377.5 & MS \\
\hline BL 1375 & 24 & 45 & 58 & 430.0 & MS & 24 & 45 & 58 & 430.0 & MS \\
\hline BL 1378 & 22 & 45 & 59 & 422.5 & MS & 22 & 45 & 58 & 422.5 & MS \\
\hline BL 1390 & 25 & 45 & 65 & 450.0 & MS & 25 & 45 & 65 & 450.0 & MS \\
\hline BL 1397 & 23 & 34 & 45 & 340.0 & MR & 13 & 34 & 45 & 315.0 & MR \\
\hline BL 1400 & 13 & 25 & 37 & 250.0 & MR & 2 & 25 & 37 & 222.5 & MR \\
\hline BL 1403 & 23 & 44 & 58 & 417.5 & MS & 23 & 44 & 56 & 417.5 & MS \\
\hline BL 1404 & 24 & 46 & 58 & 435.0 & MS & 24 & 46 & 58 & 432.5 & MS \\
\hline BL 1411 & 25 & 47 & 59 & 445.0 & MS & 25 & 47 & 58 & 442.5 & MS \\
\hline BL 1413 & 12 & 34 & 58 & 364.0 & MS & 12 & 34 & 58 & 342.5 & MS \\
\hline BL 1416 & 12 & 25 & 47 & 272.5 & MR & 12 & 24 & 47 & 267.5 & MR \\
\hline BL 1420 & 13 & 37 & 59 & 365.0 & MS & 13 & 37 & 58 & 362.5 & MS \\
\hline BL 1421 & 23 & 37 & 58 & 387.5 & MS & 23 & 36 & 58 & 382.5 & MS \\
\hline BL 1429 & 24 & 45 & 58 & 435.0 & MS & 24 & 45 & 58 & 425.0 & MS \\
\hline BL 1430 & 13 & 36 & 58 & 365.0 & MS & 13 & 36 & 58 & 357.5 & MS \\
\hline BL 1440 & 22 & 35 & 58 & 370.0 & MS & 22 & 35 & 58 & 372.5 & MS \\
\hline BL 1443 & 22 & 35 & 58 & 362.5 & MS & 23 & 37 & 58 & 365.0 & MS \\
\hline BL 1451 & 23 & 45 & 58 & 425.0 & MS & 23 & 46 & 58 & 430.0 & MS \\
\hline BL 1470 & 13 & 37 & 58 & 364.5 & MS & 13 & 36 & 58 & 362.5 & MS \\
\hline BL 1473 & 13 & 35 & 58 & 367.5 & MS & 13 & 35 & 58 & 372.5 & MS \\
\hline BL 1475 & 34 & 46 & 67 & 482.5 & MS & 34 & 46 & 67 & 482.5 & MS \\
\hline BL 1500 & 25 & 46 & 79 & 490.0 & $\mathrm{~S}$ & 25 & 46 & 78 & 487.5 & $\mathrm{~S}$ \\
\hline BL 1501 & 25 & 45 & 67 & 455.0 & MS & 25 & 45 & 67 & 455.0 & MS \\
\hline
\end{tabular}




\begin{tabular}{|c|c|c|c|c|c|c|c|c|c|c|}
\hline BL 1502 & 33 & 45 & 59 & 455.0 & MS & 33 & 45 & 58 & 452.5 & MS \\
\hline BL 1503 & 22 & 34 & 58 & 365.0 & MS & 22 & 34 & 58 & 370.0 & MS \\
\hline BL 1504 & 12 & 24 & 36 & 240.0 & MR & 12 & 24 & 35 & 237.5 & MR \\
\hline BL 1505 & 24 & 36 & 58 & 382.5 & MS & 24 & 36 & 58 & 382.5 & MS \\
\hline BL 1506 & 23 & 44 & 58 & 420.0 & MS & 23 & 44 & 58 & 422.5 & MS \\
\hline BL 1507 & 13 & 36 & 58 & 357.5 & MS & 13 & 36 & 58 & 365.0 & MS \\
\hline BL 1508 & 22 & 45 & 67 & 447.5 & MS & 22 & 45 & 67 & 447.5 & MS \\
\hline BL 1509 & 24 & 37 & 58 & 390.0 & MS & 24 & 37 & 58 & 390.0 & MS \\
\hline BL 1510 & 34 & 46 & 58 & 460.0 & MS & 34 & 46 & 59 & 457.5 & MS \\
\hline BL 1511 & 22 & 34 & 58 & 367.5 & MS & 22 & 34 & 58 & 370.0 & MS \\
\hline BL 1512 & 24 & 46 & 58 & 435.0 & MS & 24 & 46 & 58 & 432.5 & MS \\
\hline BL 1513 & 32 & 44 & 58 & 440.0 & MS & 32 & 44 & 58 & 440.0 & MS \\
\hline BL 1514 & 13 & 36 & 58 & 357.5 & MS & 13 & 36 & 58 & 357.5 & MS \\
\hline BL 1515 & 22 & 35 & 58 & 370.0 & MS & 22 & 34 & 58 & 365.0 & MS \\
\hline BL 1516 & 13 & 24 & 48 & 272.5 & MR & 13 & 24 & 46 & 267.5 & MR \\
\hline BL 1517 & 13 & 26 & 47 & 280.0 & MR & 13 & 26 & 47 & 280.0 & MR \\
\hline BL 1518 & 23 & 34 & 46 & 342.5 & MR & 23 & 34 & 46 & 342.5 & MR \\
\hline BL 1519 & 22 & 35 & 58 & 375.0 & MS & 22 & 35 & 58 & 370.0 & MS \\
\hline BL 1520 & 22 & 34 & 45 & 337.5 & MR & 22 & 34 & 46 & 340.0 & MR \\
\hline BL 1521 & 33 & 45 & 58 & 452.5 & MS & 33 & 45 & 58 & 452.5 & MS \\
\hline BL 1522 & 13 & 34 & 65 & 365.0 & MS & 13 & 34 & 58 & 364.5 & MS \\
\hline BL 1523 & 12 & 34 & 58 & 410.0 & MS & 22 & 46 & 58 & 410.0 & MS \\
\hline BL 1524 & 12 & 35 & 58 & 365.0 & MS & 12 & 36 & 58 & 365.0 & MS \\
\hline BL 1525 & 13 & 34 & 68 & 372.5 & MS & 13 & 34 & 67 & 370.0 & MS \\
\hline BL 1526 & 23 & 36 & 58 & 380.0 & MS & 23 & 34 & 58 & 370.0 & MS \\
\hline BL 1527 & 13 & 25 & 37 & 250.0 & MR & 13 & 24 & 37 & 245.0 & MR \\
\hline BL 1528 & 22 & 44 & 58 & 420.0 & MS & 22 & 36 & 58 & 380.0 & MS \\
\hline BL 1529 & 12 & 34 & 58 & 362.5 & MS & 12 & 34 & 58 & 364.5 & MS \\
\hline BL 1530 & 23 & 35 & 67 & 400.0 & MS & 23 & 35 & 67 & 400.0 & MS \\
\hline BL 1531 & 13 & 44 & 59 & 395.0 & MS & 13 & 34 & 58 & 365.0 & MS \\
\hline BL 1532 & 11 & 23 & 24 & 202.5 & $\mathrm{R}$ & 1 & 23 & 24 & 177.5 & $\mathrm{R}$ \\
\hline BL 1533 & 23 & 36 & 58 & 382.5 & MS & 23 & 36 & 58 & 380.0 & MS \\
\hline BL 1534 & 25 & 37 & 67 & 415.0 & MS & 25 & 37 & 68 & 417.5 & MS \\
\hline BL 1535 & 24 & 46 & 68 & 460.0 & MS & 24 & 46 & 67 & 457.5 & MS \\
\hline BL 1536 & 23 & 45 & 58 & 425.0 & MS & 23 & 45 & 58 & 425.0 & MS \\
\hline BL 1537 & 22 & 34 & 58 & 370.0 & MS & 22 & 34 & 58 & 370.0 & MS \\
\hline BL 1538 & 13 & 34 & 67 & 370.0 & MS & 12 & 34 & 67 & 367.5 & MS \\
\hline BL 1539 & 22 & 45 & 58 & 425.0 & MS & 22 & 35 & 58 & 375.0 & MS \\
\hline BL 1540 & 25 & 57 & 79 & 545.0 & $\mathrm{~S}$ & 25 & 57 & 79 & 545.0 & $\mathrm{~S}$ \\
\hline BL 1541 & 22 & 56 & 67 & 502.5 & MS & 22 & 56 & 68 & 505.0 & MS \\
\hline BL 1542 & 34 & 56 & 78 & 560.0 & $\mathrm{~S}$ & 34 & 56 & 78 & 560.0 & $\mathrm{~S}$ \\
\hline BL 1543 & 23 & 35 & 67 & 400.0 & MS & 23 & 35 & 67 & 400.0 & MS \\
\hline BL 1544 & 11 & 36 & 58 & 365.0 & MS & 11 & 24 & 58 & 290.0 & MS \\
\hline BL 1545 & 22 & 34 & 58 & 370.0 & MS & 12 & 34 & 58 & 365.0 & MS \\
\hline BL 1546 & 25 & 47 & 69 & 470.0 & MS & 25 & 47 & 68 & 467.5 & MS \\
\hline BL 1547 & 22 & 34 & 58 & 362.5 & MS & 22 & 34 & 58 & 365.0 & MS \\
\hline BL 1548 & 24 & 45 & 58 & 427.5 & MS & 13 & 34 & 58 & 362.0 & MS \\
\hline BL 1549 & 13 & 23 & 46 & 262.5 & MR & 13 & 24 & 46 & 267.5 & MR \\
\hline BL 1550 & 14 & 35 & 67 & 377.5 & MS & 13 & 35 & 67 & 375.0 & MS \\
\hline BL 1551 & 12 & 35 & 58 & 365.0 & MS & 12 & 35 & 58 & 367.5 & MS \\
\hline BL 1552 & 22 & 34 & 58 & 367.5 & MS & 11 & 33 & 58 & 362.5 & MS \\
\hline BL 1553 & 23 & 45 & 67 & 450.0 & MS & 23 & 45 & 67 & 450.0 & MS \\
\hline BL 1554 & 13 & 37 & 59 & 365.0 & MS & 23 & 35 & 59 & 370.0 & MS \\
\hline BL 1555 & 23 & 37 & 58 & 387.5 & MS & 23 & 35 & 58 & 377.5 & MS \\
\hline BL 1556 & 24 & 45 & 58 & 425.0 & MS & 24 & 35 & 58 & 375.0 & MS \\
\hline BL 1557 & 12 & 23 & 46 & 260.0 & MR & 12 & 23 & 46 & 260.0 & MR \\
\hline BL 1558 & 12 & 25 & 45 & 267.5 & MR & 12 & 25 & 45 & 267.5 & MR \\
\hline
\end{tabular}




\begin{tabular}{|c|c|c|c|c|c|c|c|c|c|c|}
\hline BL 1559 & 13 & 35 & 58 & 363.0 & MS & 12 & 35 & 58 & 367.5 & MS \\
\hline BL 1560 & 13 & 35 & 58 & 367.5 & MS & 13 & 35 & 58 & 363.0 & MS \\
\hline BL 1561 & 23 & 44 & 67 & 445.0 & MS & 23 & 44 & 67 & 445.0 & MS \\
\hline BL 1562 & 11 & 12 & 23 & 145.0 & $\mathrm{R}$ & 1 & 12 & 23 & 120.0 & $\mathrm{R}$ \\
\hline BL 1563 & 23 & 36 & 58 & 380.0 & MS & 23 & 36 & 58 & 380.0 & MS \\
\hline BL 1564 & 13 & 36 & 58 & 357.5 & MS & 13 & 36 & 58 & 365.0 & MS \\
\hline BL 1565 & 23 & 34 & 46 & 342.5 & MR & 23 & 34 & 46 & 342.5 & MR \\
\hline BL 1566 & 24 & 38 & 58 & 395.0 & MS & 24 & 36 & 58 & 385.0 & MS \\
\hline BL 1567 & 22 & 33 & 58 & 362.0 & MS & 22 & 34 & 58 & 365.0 & MS \\
\hline BL 1568 & 24 & 46 & 58 & 435.0 & MS & 24 & 46 & 58 & 435.0 & MS \\
\hline BL 1569 & 32 & 44 & 58 & 440.0 & MS & 32 & 44 & 59 & 440.0 & MS \\
\hline BL 1570 & 13 & 35 & 58 & 362.5 & MS & 13 & 35 & 58 & 352.5 & MS \\
\hline BL 1571 & 23 & 35 & 58 & 375.0 & MS & 23 & 35 & 57 & 375.0 & MS \\
\hline BL 1572 & 22 & 37 & 58 & 367.5 & MS & 13 & 24 & 57 & 295.0 & MS \\
\hline BL 1573 & 24 & 35 & 67 & 402.5 & MS & 24 & 35 & 67 & 402.5 & MS \\
\hline BL 1574 & 23 & 45 & 68 & 452.5 & MS & 23 & 45 & 68 & 452.5 & MS \\
\hline BL 1575 & 22 & 43 & 58 & 410.0 & MS & 22 & 35 & 58 & 370.0 & MS \\
\hline BL 1576 & 25 & 57 & 79 & 545.0 & $\mathrm{~S}$ & 25 & 57 & 79 & 545.0 & $\mathrm{~S}$ \\
\hline BL 1577 & 34 & 46 & 67 & 482.5 & MS & 34 & 46 & 67 & 482.5 & MS \\
\hline BL 1578 & 13 & 34 & 58 & 365.0 & MS & 13 & 34 & 58 & 347.5 & MS \\
\hline BL 1579 & 13 & 35 & 58 & 367.5 & MS & 13 & 35 & 58 & 363.0 & MS \\
\hline BL 1580 & 13 & 37 & 59 & 365.0 & MS & 13 & 37 & 59 & 365.0 & MS \\
\hline BL 1581 & 23 & 34 & 58 & 372.5 & MS & 23 & 37 & 58 & 377.5 & MS \\
\hline BL 1582 & 22 & 35 & 58 & 365.0 & MS & 22 & 24 & 58 & 367.5 & MS \\
\hline BL 1583 & 22 & 245 & 58 & 362.5 & MS & 22 & 35 & 58 & 362.5 & MS \\
\hline BL 1584 & 13 & 36 & 67 & 380.0 & MS & 13 & 36 & 67 & 380.0 & MS \\
\hline BL 1585 & 12 & 24 & 46 & 265.0 & MR & 12 & 24 & 46 & 265.0 & MR \\
\hline BL 1586 & 24 & 35 & 58 & 380.0 & MS & 24 & 36 & 58 & 385.0 & MS \\
\hline BL 1587 & 23 & 36 & 59 & 368.0 & MS & 13 & 36 & 58 & 365.0 & MS \\
\hline BL 1588 & 25 & 37 & 58 & 392.5 & MS & 25 & 37 & 58 & 392.5 & MS \\
\hline BL 1589 & 23 & 35 & 57 & 375.0 & MS & 23 & 35 & 58 & 375.0 & MS \\
\hline BL 1590 & 24 & 36 & 47 & 357.5 & MR & 24 & 36 & 47 & 357.5 & MR \\
\hline BL 1591 & 23 & 36 & 58 & 382.5 & MS & 23 & 35 & 58 & 377.5 & MS \\
\hline BL 1592 & 25 & 36 & 58 & 387.5 & MS & 25 & 36 & 58 & 387.5 & MS \\
\hline BL 1593 & 22 & 35 & 67 & 397.5 & MS & 11 & 35 & 67 & 370.0 & MS \\
\hline BL 1594 & 33 & 44 & 67 & 470.0 & MS & 23 & 44 & 67 & 445.0 & MS \\
\hline BL 1595 & 25 & 46 & 68 & 462.5 & MS & 25 & 46 & 68 & 462.5 & MS \\
\hline BL 1596 & 22 & 34 & 58 & 370.0 & MS & 22 & 34 & 58 & 370.0 & MS \\
\hline BL 1597 & 23 & 24 & 36 & 267.5 & MR & 23 & 24 & 35 & 265.0 & MR \\
\hline BL 1598 & 22 & 34 & 58 & 372.5 & MS & 22 & 34 & 58 & 365.0 & MS \\
\hline BL 1599 & 24 & 45 & 58 & 427.5 & MS & 24 & 45 & 58 & 427.5 & MS \\
\hline BL 1600 & 24 & 46 & 58 & 435.0 & MS & 24 & 46 & 58 & 435.0 & MS \\
\hline BL 1601 & 22 & 45 & 59 & 422.5 & MS & 13 & 45 & 58 & 395.0 & MS \\
\hline BL 1602 & 25 & 45 & 66 & 452.5 & MS & 25 & 46 & 66 & 457.5 & MS \\
\hline BL 1603 & 23 & 44 & 58 & 417.5 & MS & 23 & 45 & 58 & 422.5 & MS \\
\hline BL 1604 & 24 & 46 & 58 & 435.0 & MS & 24 & 46 & 58 & 435.0 & MS \\
\hline BL 1605 & 25 & 47 & 58 & 442.5 & MS & 25 & 47 & 58 & 440.0 & MS \\
\hline BL 1606 & 13 & 37 & 58 & 362.5 & MS & 13 & 37 & 58 & 365.0 & MS \\
\hline BL 1607 & 23 & 37 & 59 & 390.0 & MS & 23 & 37 & 59 & 390.0 & MS \\
\hline BL 1608 & 24 & 45 & 59 & 427.5 & MS & 24 & 46 & 58 & 432.5 & MS \\
\hline BL 1609 & 13 & 36 & 58 & 365.0 & MS & 13 & 36 & 58 & 365.0 & MS \\
\hline BL 1610 & 13 & 37 & 59 & 367.5 & MS & 13 & 35 & 58 & 370.0 & MS \\
\hline BL 1611 & 23 & 35 & 58 & 377.5 & MS & 23 & 35 & 58 & 377.5 & MS \\
\hline BL 1612 & 34 & 46 & 67 & 482.5 & MS & 34 & 46 & 67 & 482.5 & MS \\
\hline BL 1613 & 12 & 26 & 57 & 302.5 & MS & 23 & 45 & 58 & 377.5 & MS \\
\hline BL 1614 & 25 & 45 & 67 & 455.0 & MS & 25 & 45 & 67 & 455.0 & MS \\
\hline BL 1615 & 33 & 45 & 59 & 455.0 & MS & 33 & 45 & 58 & 452.5 & MS \\
\hline
\end{tabular}




\begin{tabular}{|c|c|c|c|c|c|c|c|c|c|c|}
\hline BL 1616 & 24 & 45 & 58 & 427.5 & MS & 24 & 45 & 58 & 427.5 & MS \\
\hline BL 1617 & 23 & 45 & 68 & 452.5 & MS & 23 & 45 & 68 & 452.5 & MS \\
\hline BL 1618 & 13 & 36 & 58 & 357.5 & MS & 13 & 36 & 58 & 365.0 & MS \\
\hline BL 1619 & 22 & 45 & 67 & 447.5 & MS & 22 & 45 & 67 & 447.5 & MS \\
\hline BL 1620 & 24 & 37 & 58 & 390.0 & MS & 23 & 37 & 58 & 387.5 & MS \\
\hline BL 1621 & 34 & 46 & 58 & 460.0 & MS & 34 & 46 & 58 & 460.0 & MS \\
\hline BL 1622 & 22 & 35 & 58 & 372.5 & MS & 23 & 35 & 58 & 375.0 & MS \\
\hline BL 1623 & 24 & 46 & 68 & 460.0 & MS & 24 & 46 & 68 & 460.0 & MS \\
\hline BL 1624 & 32 & 44 & 58 & 440.0 & MS & 32 & 44 & 59 & 442.5 & MS \\
\hline BL 1625 & 13 & 37 & 59 & 365.0 & MS & 13 & 36 & 59 & 362.0 & MS \\
\hline BL 1626 & 13 & 35 & 58 & 370.0 & MS & 23 & 35 & 59 & 368.0 & MS \\
\hline BL 1627 & 25 & 36 & 58 & 385.0 & MS & 25 & 36 & 58 & 385.0 & MS \\
\hline BL 1628 & 23 & 35 & 58 & 377.5 & MS & 23 & 35 & 58 & 375.0 & MS \\
\hline BL 1629 & 24 & 36 & 58 & 385.0 & MS & 24 & 36 & 58 & 385.0 & MS \\
\hline BL 1630 & 25 & 35 & 58 & 380.0 & MS & 25 & 35 & 58 & 380.0 & MS \\
\hline BL 1631 & 22 & 36 & 58 & 380.0 & MS & 11 & 36 & 58 & 362.5 & MS \\
\hline BL 1632 & 33 & 35 & 67 & 425.0 & MS & 33 & 35 & 67 & 425.0 & MS \\
\hline BL 1633 & 24 & 37 & 58 & 390.0 & MS & 24 & 37 & 58 & 390.0 & MS \\
\hline BL 1634 & 25 & 47 & 69 & 470.0 & MS & 25 & 47 & 68 & 467.5 & MS \\
\hline BL 1635 & 22 & 34 & 58 & 365.0 & MS & 22 & 34 & 58 & 370.0 & MS \\
\hline BL 1636 & 24 & 45 & 58 & 427.5 & MS & 24 & 45 & 59 & 427.5 & MS \\
\hline BL 1637 & 23 & 45 & 58 & 427.5 & MS & 13 & 45 & 58 & 402.5 & MS \\
\hline BL 1638 & 22 & 46 & 59 & 427.5 & MS & 22 & 46 & 59 & 427.5 & MS \\
\hline BL 1639 & 25 & 45 & 67 & 455.0 & MS & 25 & 45 & 68 & 457.5 & MS \\
\hline BL 1640 & 23 & 44 & 58 & 417.5 & MS & 23 & 46 & 58 & 422.5 & MS \\
\hline BL 1641 & 25 & 47 & 59 & 445.0 & MS & 25 & 47 & 59 & 445.0 & MS \\
\hline BL 1642 & 13 & 36 & 58 & 357.5 & MS & 13 & 36 & 58 & 367.5 & MS \\
\hline BL 1643 & 24 & 45 & 58 & 427.5 & MS & 24 & 45 & 58 & 425.0 & MS \\
\hline BL 1644 & 13 & 36 & 58 & 365.0 & MS & 13 & 36 & 58 & 365.0 & MS \\
\hline BL 1645 & 13 & 36 & 58 & 367.5 & MS & 23 & 37 & 59 & 372.5 & MS \\
\hline BL 1646 & 34 & 46 & 67 & 482.5 & MS & 34 & 46 & 67 & 482.5 & MS \\
\hline BL 1647 & 25 & 44 & 67 & 450.0 & MS & 25 & 44 & 68 & 452.5 & MS \\
\hline BL 1648 & 33 & 45 & 59 & 455.0 & MS & 33 & 45 & 58 & 452.5 & MS \\
\hline BL 1649 & 23 & 45 & 66 & 447.5 & MS & 23 & 46 & 66 & 452.5 & MS \\
\hline BL 1650 & 13 & 36 & 58 & 364.5 & MS & 13 & 36 & 58 & 352.5 & MS \\
\hline BL 1651 & 12 & 36 & 58 & 362.5 & MS & 12 & 34 & 59 & 342.5 & MS \\
\hline BL 1652 & 24 & 56 & 78 & 535.0 & $\mathrm{~S}$ & 24 & 56 & 78 & 535.0 & $\mathrm{~S}$ \\
\hline BL 1653 & 25 & 47 & 68 & 467.5 & MS & 24 & 47 & 68 & 465.0 & MS \\
\hline BL1654 & 24 & 45 & 58 & 427.5 & MS & 25 & 46 & 58 & 435.0 & MS \\
\hline BL 1655 & 22 & 45 & 58 & 422.5 & MS & 22 & 45 & 58 & 425.0 & MS \\
\hline BL 1656 & 23 & 46 & 58 & 422.5 & MS & 23 & 45 & 56 & 422.5 & MS \\
\hline BL 1657 & 13 & 36 & 59 & 345.0 & MS & 13 & 34 & 58 & 365.0 & MS \\
\hline BL 1658 & 23 & 24 & 46 & 292.5 & MR & 23 & 34 & 46 & 342.5 & MR \\
\hline BL 1659 & 13 & 37 & 58 & 362.5 & MS & 13 & 37 & 58 & 362.5 & MS \\
\hline BL 1660 & 13 & 36 & 58 & 365.0 & MS & 13 & 36 & 58 & 355.0 & MS \\
\hline BL 1661 & 23 & 34 & 58 & 372.5 & MS & 23 & 34 & 56 & 367.5 & MS \\
\hline BL 1662 & 13 & 37 & 59 & 365.0 & MS & 13 & 37 & 58 & 362.0 & MS \\
\hline BL 1663 & 24 & 45 & 57 & 427.5 & MS & 24 & 46 & 59 & 432.5 & MS \\
\hline BL 1664 & 23 & 36 & 58 & 382.5 & MS & 23 & 36 & 58 & 382.5 & MS \\
\hline BL 1665 & 22 & 36 & 58 & 362.5 & MS & 22 & 45 & 58 & 370.0 & MS \\
\hline BL 1666 & 22 & 25 & 48 & 300.0 & MR & 22 & 25 & 47 & 297.5 & MR \\
\hline BL 1667 & 13 & 37 & 59 & 365.0 & MS & 13 & 34 & 58 & 365.0 & MS \\
\hline BL 1668 & 23 & 44 & 67 & 445.0 & MS & 23 & 44 & 67 & 445.0 & MS \\
\hline BL 1669 & 13 & 36 & 58 & 367.5 & MS & 13 & 36 & 58 & 377.5 & MS \\
\hline BL 1670 & 22 & 45 & 67 & 447.5 & MS & 22 & 45 & 67 & 447.5 & MS \\
\hline BL 1671 & 22 & 34 & 58 & 367.5 & MS & 12 & 34 & 59 & 372.5 & MS \\
\hline BL 1672 & 13 & 34 & 58 & 365.5 & MS & 13 & 34 & 58 & 367.5 & MS \\
\hline
\end{tabular}




\begin{tabular}{|c|c|c|c|c|c|c|c|c|c|c|}
\hline BL 1673 & 22 & 36 & 58 & 380.0 & MS & 22 & 36 & 59 & 377.5 & MS \\
\hline BL 1674 & 23 & 35 & 58 & 375.0 & MS & 23 & 35 & 58 & 377.5 & MS \\
\hline IBYT-18-4 & 24 & 35 & 58 & 375.0 & MS & 24 & 35 & 58 & 375.0 & MS \\
\hline IBYT-18-5 & 13 & 36 & 58 & 370.0 & MS & 13 & 36 & 59 & 365.0 & MS \\
\hline IBYT - 18-6 & 25 & 37 & 58 & 392.5 & MS & 25 & 37 & 58 & 392.5 & MS \\
\hline IBYT-18-8 & 23 & 35 & 59 & 375.0 & MS & 23 & 35 & 58 & 377.5 & MS \\
\hline IBYT-18-9 & 24 & 36 & 48 & 360.0 & MR & 24 & 36 & 48 & 360.0 & MR \\
\hline IBYT-18-12 & 24 & 36 & 59 & 387.5 & MS & 24 & 36 & 58 & 365.0 & MS \\
\hline IBYT-18-16 & 25 & 36 & 58 & 387.5 & MS & 25 & 36 & 57 & 385.0 & MS \\
\hline IBYT-18-18 & 24 & 37 & 59 & 387.5 & MS & 24 & 37 & 68 & 415.0 & MS \\
\hline IBYT-18-21 & 22 & 36 & 58 & 380.0 & MS & 22 & 36 & 58 & 380.0 & MS \\
\hline INBYT-HI-18-3 & 33 & 44 & 67 & 470.0 & MS & 33 & 45 & 67 & 475.0 & MS \\
\hline INBYT-HI-18-9 & 24 & 37 & 58 & 390.0 & MS & 23 & 37 & 58 & 387.5 & MS \\
\hline INBYT-HI-18-11 & 13 & 26 & 47 & 280.0 & MR & 13 & 26 & 47 & 280.0 & MR \\
\hline INBYT-HI-18-13 & 25 & 47 & 69 & 470.0 & MS & 25 & 47 & 69 & 470.0 & MS \\
\hline INBYT-HI-18-18 & 22 & 35 & 58 & 362.5 & MS & 12 & 36 & 59 & 364.0 & MS \\
\hline INBYT-HI-18-22 & 24 & 45 & 58 & 427.5 & MS & 24 & 45 & 58 & 427.5 & MS \\
\hline 5thGSBYT-18-3 & 24 & 46 & 58 & 435.0 & MS & 24 & 46 & 58 & 435.0 & MS \\
\hline 5thGSBYT-18-4 & 22 & 45 & 59 & 422.5 & MS & 22 & 46 & 59 & 427.5 & MS \\
\hline 5thGSBYT-18-6 & 25 & 45 & 65 & 450.0 & MS & 25 & 45 & 65 & 450.0 & MS \\
\hline 5thGSBYT18-7 & 23 & 44 & 59 & 417.5 & MS & 23 & 46 & 59 & 425.0 & MS \\
\hline 5thGSBYT-18-15 & 24 & 46 & 58 & 435.0 & MS & 24 & 46 & 58 & 435.0 & MS \\
\hline 5thGSBYT-18-16 & 25 & 47 & 59 & 445.0 & MS & 25 & 47 & 59 & 445.0 & MS \\
\hline 5thGSBYT-18-19 & 23 & 36 & 47 & 355.0 & MR & 13 & 36 & 47 & 330.0 & MR \\
\hline 5thGSBYT-18-21 & 13 & 37 & 59 & 365.0 & MS & 13 & 36 & 59 & 365.0 & MS \\
\hline 5thGSBYT-18-22 & 23 & 37 & 58 & 387.5 & MS & 23 & 37 & 58 & 387.5 & MS \\
\hline IBON-18-46 & 24 & 45 & 58 & 427.5 & MS & 23 & 45 & 58 & 425.0 & MS \\
\hline IBON-18-47 & 12 & 23 & 45 & 257.5 & MR & 12 & 23 & 46 & 260.0 & MR \\
\hline IBON-18-59 & 13 & 36 & 57 & 365.0 & MS & 13 & 36 & 58 & 365.0 & MS \\
\hline IBON-18-60 & 12 & 25 & 45 & 267.5 & MR & 12 & 24 & 45 & 262.5 & MR \\
\hline IBON-18-82 & 23 & 36 & 58 & 377.5 & MS & 13 & 36 & 58 & 368.5 & MS \\
\hline IBON-18-97 & 34 & 46 & 67 & 482.5 & MS & 34 & 46 & 67 & 482.5 & MS \\
\hline IBON-18-100 & 12 & 26 & 46 & 275.0 & MR & 12 & 25 & 46 & 270.0 & MR \\
\hline IBON-18-108 & 25 & 46 & 67 & 460.0 & MS & 25 & 46 & 68 & 462.5 & MS \\
\hline INBON-HI-18-7 & 33 & 45 & 59 & 455.0 & MS & 33 & 45 & 58 & 452.5 & MS \\
\hline INBON-HI-18-11 & 24 & 36 & 58 & 382.5 & MS & 13 & 36 & 58 & 365.0 & MS \\
\hline INBON-HI-18-26 & 23 & 45 & 68 & 452.5 & MS & 23 & 45 & 67 & 450.0 & MS \\
\hline INBON-HI-18-48 & 13 & 36 & 58 & 367.5 & MS & 13 & 36 & 58 & 367.5 & MS \\
\hline INBON-HI-18-49 & 22 & 45 & 67 & 447.5 & MS & 22 & 45 & 67 & 447.5 & MS \\
\hline INBON-HI-18-55 & 24 & 37 & 58 & 390.0 & MS & 24 & 36 & 59 & 382.5 & MS \\
\hline 5thGSBON-18-65 & 34 & 46 & 68 & 485.0 & MS & 34 & 46 & 68 & 485.0 & MS \\
\hline 5thGSBON-18-79 & 22 & 34 & 58 & 367.5 & MS & 22 & 34 & 58 & 367.5 & MS \\
\hline 5thGSBON-18-84 & 24 & 46 & 68 & 460.0 & MS & 14 & 46 & 68 & 435.0 & MS \\
\hline 5thGSBON-18-94 & 32 & 44 & 58 & 440.0 & MS & 32 & 44 & 58 & 442.5 & MS \\
\hline 5thGSBON-18-104 & 13 & 37 & 59 & 365.0 & MS & 13 & 37 & 58 & 362.5 & MS \\
\hline DWRUB 52 & 23 & 35 & 47 & 350.0 & MR & 23 & 35 & 47 & 350.0 & MR \\
\hline DWRB-92 & 34 & 46 & 58 & 460.0 & MS & 34 & 46 & 58 & 460.0 & MS \\
\hline DWRB-123 & 23 & 36 & 46 & 352.5 & MR & 23 & 36 & 47 & 355.0 & MR \\
\hline PL-807 & 24 & 36 & 47 & 357.5 & MR & 24 & 35 & 47 & 352.5 & MR \\
\hline PL-891 & 1 & 12 & 13 & 95.0 & $\mathrm{R}$ & 1 & 12 & 23 & 120.0 & $\mathrm{R}$ \\
\hline ВH 902 & 15 & 26 & 37 & 260.0 & MR & 15 & 26 & 37 & 260.0 & MR \\
\hline ВH 946 & 13 & 25 & 36 & 247.5 & MR & 13 & 25 & 36 & 247.5 & MR \\
\hline RD 2849 & 26 & 37 & 38 & 345.0 & MR & 26 & 37 & 38 & 345.0 & MR \\
\hline RD 2917 & 13 & 25 & 38 & 252.5 & MR & 13 & 25 & 37 & 250.0 & MR \\
\hline PL-426 & 56 & 78 & 89 & 752.5 & $\mathrm{~S}$ & 46 & 78 & 89 & 727.5 & $\mathrm{~S}$ \\
\hline
\end{tabular}

*F- Flowering, D- Dough, HD- Hard Dough stage; ** DS- Disease Reaction 
Table.3 Categorization of barley entries based on their disease reaction during two cropping seasons (2017-18 and 2018-19)

\begin{tabular}{|c|c|c|c|c|}
\hline $\begin{array}{c}\text { Range } \\
\text { of value } \\
\text { (DD)* }\end{array}$ & $\begin{array}{l}\text { Disease } \\
\text { reaction }\end{array}$ & $\begin{array}{l}\text { Range of } \\
\text { AUDPC }\end{array}$ & Barley entries & $\begin{array}{c}\text { Total } \\
\text { no. of } \\
\text { entries }\end{array}$ \\
\hline 00-13 & $\begin{array}{l}\text { Highly } \\
\text { resistant }\end{array}$ & 0.00 & -NIL- & 0 \\
\hline 14-35 & Resistant & $1-180$ & BL-1309, BL-1313, BL-1532, BL- 1562 and PL-891 & 5 \\
\hline 36-57 & $\begin{array}{l}\text { Moderately } \\
\text { Resistant }\end{array}$ & $181-360$ & $\begin{array}{l}\text { BL-1314, BL-1325, BL-1397, BL-1400, BL-1416, BL-1504, BL-1516, BL- } \\
\text { 1517, BL-1518, BL-1520, BL-1527,BL-1549, BL-1557, BL-1558, BL- } \\
\text { 1565, BL-1585, BL-1590, BL-1597, BL-1658, BL-1666, IBYT-18-9, } \\
\text { INBYT-HI-18-11, 5thGSBYT-18-19, IBON-18-47, IBON-18-60, IBON- } \\
\text { 18-100, DWRUB-52, DWRB-123, PL-807, BH-902, BH-946, RD-2849, } \\
\text { RD-2917, }\end{array}$ & 33 \\
\hline 58-69 & $\begin{array}{l}\text { Moderately } \\
\text { Susceptible }\end{array}$ & $361-500$ & $\begin{array}{l}\text { BL-1301, BL-1319, BL-1322, BL-1335, BL-1338, BL-1340, BL-1363, BL- } \\
\text { 1367, BL-1368, BL-1369, BL-1375, BL-1378, BL-1390, BL-1403, BL- } \\
\text { 1404, Bl-1411, BL-1413, BL-1420, BL-1421, BL-1429, BL-1430, B1-1440, } \\
\text { BL-1443, BL-1451, BL-1470, BL-1473, BL-1475, bl-1500, BL-1501, BL- } \\
\text { 1502, BL-1503, BL-1505, BL-1506, BL-1507, BL1508, BL-1509, BL- } \\
\text { 1510, BL-1511, BL-1512, BL-1513, BL-1514, BL-1515, BL-1519, BL- } \\
\text { 1521, BL-1522, BL- 1523, BL-1524, BL-1525, BL-1526, BL-1528, BL- } \\
\text { 1529 BL-1530, BL-1531, BL-1533, BL-1534, BL-1535, BL-1536, BL- } \\
\text { 1537, BL-1538, BL-1539, BL-1541, BL-1543, BL-1544, BL-1545, BL- } \\
\text { 1546, BL-1547, BL-1548,BL-1550, BL-1551, BL-1552, BL-1553, BL- } \\
\text { 1554, BL-1555, BL-1556, BL-1559, BL-1560, BL-1561, BL-1563, BL- } \\
\text { 1564, BL-1566, BL-1567, BL-1568, BL-1569, BL-1570, BL-1571, BL- } \\
\text { 1572, BL-1573, BL-1574, BL-1575, BL-1577, BL-1578, BL-1579, BL- } \\
\text { 1580, BL-1581, BL-1582, BL-1583, BL-1584, BL-1586, BL-1587, BL- } \\
\text { 1588, BL-1589, BL-1591, BL-1592, BL-1593, BL-1594, BL-1595, BL- } \\
\text { 1596, BL-1598, BL-1599, BL-1600, BL- 1601, BL-1602, BL-1603, BL- } \\
\text { 1604, BL-1605, BL-1606, BL-1607, BL-1608, BL-1609, BL-1610, BL- } \\
\text { 1611, BL-1612, BL-1613, BL-1614, BL-1616, BL-1617, BL-1618, BL- } \\
\text { 1619, BL-1620, BL-1621, BL-1622, BL-1623, BL-1624, BL-1625, BL- } \\
\text { 1626, BL-1627, BL-1628, BL-1629, BL-1630, BL-1631, BL-1632, BL- } \\
\text { 1633, BL-1634, BL-1635, BL-1636, BL-1637, BL-1638, BL-1639, BL- } \\
\text { 1640, BL-1641, BL-1642, BL-1643, BL-1644, BL-1645, BL-1646, BL- } \\
\text { 1647, BL-1648, BL-1649, BL-1650, BL-1651, BL-1653, BL-1654, BL- } \\
\text { 1655, BL-1656, BL-1657, BL-1659, BL-1660, BL-1661, BL-1662, BL- } \\
\text { 1663, BL-1664, BL-1665, BL-1667, BL-1668, BL-1669, BL-1670, BL- } \\
\text { 1671, BL-1672, BL-1673, BL-1674, DWRB-92, IBYT-18-4, IBYT-18-5, } \\
\text { IBYT-18-6, IBYT-18-8, IBYT-18-12, IBYT-18-16, IBYT-18-18, IBYT-18- } \\
\text { 21, INBYT-HI-18-3, INBYT-HI-18-9, INBYT-HI-18-13, INBYT-HI-18- } \\
\text { 18, INBYT-HI-18-22, 5thGSBYT-18-3, 5thGSBYT-18-4, 5thGSBYT-18-6, } \\
\text { 5thGSBYT-18-7, 5thGSBYT-18-15, 5thGSBYT-18-16, 5thGSBYT-18-21, } \\
\text { 5thGSBYT-18-22, IBON-18-46, IBON-18-59, IBON-18-82, IBON-18-97, } \\
\text { IBON-18-108, INBON-HI-18-7, INBON-HI-18-11, INBON-HI-18-26, } \\
\text { INBON-HI-18-48, INBON-HI-18-49, INBON-HI-18-55, 5thGSBON-18- } \\
\text { 65, 5thGSBON-18-79, 5thGSBON-18-84, 5thGSBON-18-94, 5thGSBON- } \\
\text { 18-104. }\end{array}$ & 218 \\
\hline$>69$ & Susceptible & $\begin{array}{l}500 \text { and } \\
\text { above }\end{array}$ & BL-1500, BL-1540, BL-1542, BL-1576, BL-1652, PL-426 & 6 \\
\hline
\end{tabular}

*First and second value represents percent blighted area on the flag leaf and flag-1 leaves respectively. Values 1,2,3,4,5,6,7,8, and 9 correspond to $10,20,30,40,50,60,70,80$ and 90 percent blighted area respectively 
Bipolaris sorokiniana is also known to cause foliar blight or spot blotch disease in wheat crop as well. Screening of two hundred wheat germplasm accessions against this pathogen by Latwal et al., (2016) has revealed that on the basis of their AUDPC values over two years of testing, four accessions were found to be highly resistant whereas eventy eight exhibited resistant reaction towards disease. Similarly, screening of 126 barley genotypes against $B$. sorokiniana in inner tarai region of Nepal was also carried out by Subedi et al., (2020) and among these genotypes resistance was observed in eight genotypes while, thirty two genotypes exhibited moderately resistant reaction.

Therefore, in the present findings, the barley entries resistant to spot blotch disease in their field testing for two successive seasons under artificial inoculated conditions were able to keep the disease intensity on the plant to very low level and thus can act as useful source in incorporating spot blotch resistance in high yielding cultivars of barley which are prior found to be susceptible to the disease.

\section{References}

Anonymous., 2019. Package of Practices for Rabi Crops of Punjab. pp 53 Punjab Agricultural University, Ludhiana.

Arabi, M.I.E. and Jawahar, M. 2003. Pathotypes of Cochliobolus sativus (spot blotch) on barley in Syria. Journal of Plant Pathology,85:193196.

Bala, A., andKaur, S. 2008. Cross infectivity of Bipolaris sorokiniana among wheat, barley, triticale, rye and Phalaris minor. Plant Disease Research, 23: 712.

Chaurasia, S., Joshi, A.K., Dhari, R., andChand, R. 1999. Resistance to foliar blight of wheat: a search. Genetic Resources and Crop
Evolution, 46: 469-475.

Duveiller, E., Garcia, I., Franco, J., Toledo, J., Crossa, J., and Lopez, F. 1998. Evaluating spot blotch resistance of wheat: Improving disease assessment under controlled condition and in the field. In: Helminthosporium blights of wheat: Spot blotch and Tan spot (Duveiller, E., Dubin, H.J., Reeves, J., McNab, A. eds.) Mexico. D.F., Mexico: CIMMYT. pp. 63-66.

Iftikhar, S.,Asad, S., Ratt, A.U.R., Fayya, M., and Munir,A. 2009. Selection of Barley germplasm resistant to spot blotch. Pakistan Journal of Botany,41: 309-314.

Jain, N., Malik, R., Selvakumar, R., Kumar, R., Pandey, V., and Verma, R.P.S. 2014. Screening of barley germplasm for leaf blight (Bipolaris sorokiniana) resistance. Indian J Agric Res 48: 67 71.

Kutcher, H.R., Bailey, K.L., Rossnagel, B.G., and Legge, W.G. 1994. Heritability of common root rot and spot blotch resistance in barley. Canadian Journal of Plant Pathology, 16: 287-294.

Latwal, C., Deepshikha, Kumari, B., Singh, P.K., and Jaiswal, J.P. 2016. Characterization of bread wheat germplasm for spot blotch resistance and its association with yield and yield related traits.Journal of Wheat Research, 8: 31-37.

Nutter, F.W., Pederson Jr. V.D., andFoster, A.E. 1995. Foster Effect of inoculation with Cochliobolus sativus at specific growth stages on grain yield and quality of malting barley. Crop Science, 2: 993-938.

Roelfs, A.P., Singh, R.P., and Saari, E.E. 1992.Rust diseases of wheat: Concepts and methods of disease management. CIMMYT, Mexico.

Saari, E.E., and Prescott, J.M. 1975. A scale for appraising the foliar intensity of 
wheat disease. Plant Disease Reporter 59: 377-380.

Singh, D.P., Kumar, P., andSingh, S.K. 2005. Resistance in wheat genotypes against leaf blight caused by Bipolaris sorokiniana at seedling along with adult plant stage. Indian Phytopathology, 58: 344.

Singh, D., Pandey, S.K., Singh, S.P., Singh, D.K., and Kavita. 2017. Evaluation of barley genotypes against spot botch disease caused by Bipolaris sorokiniana. Plant Archives, 17: $167-$ 170.

Singh, S.K., Singh, M., Razdan, V.K., Singh, V.B., Singh, A.K., Gupta, S., Singh, R., Gupta, A., Shankar, U., Singh, A.K., Pandey, M.K., and Sharma, R. 2018. Prevalence of spot blotch (Bipolaris sorokiniana) of wheat and its management through host resistance. International Journal of Current Microbiology and Applied Sciences, 7: 686-694.

Subedi, S., Neupane, S., Gurung, S., and Raymajhi A. Oli, 2020. Evaluation of Barley Genotypes against Spot Blotch Disease in Inner Tarai Region of Nepal. Journal of Nepal Agricultural Research Council,6: 70-78.

Taner, A., Avci, M., and Dusunceli, F. 2004. Barley post-harvest operations In: Post harvest compendium, FAO, United
States. Pp. 1-65.

Tricase, C., Amicarelli, V., Lamonaca, E., and Leonardo Rana, R. 2018. Economic Analysis of the Barley Market and Related Uses, Grasses as Food and Feed, Zerihun Tadele, IntechOpen, DOI: $\quad$ 10.5772/intechopen.78967. Available from: https://www.intechopen.com/books/gra sses-as-food-and-feed/economicanalysis-of-the-barley-market-andrelated-uses

Vaish, S.S., Bilal Ahmed, S., and Prakash, K. 2011. First documentation on status of barley diseases from the high altitude cold arid Trans-Himalayan Ladakh region of India. Crop Protection, 30: 1129-1137.

Van der Plank, J.E. 1968.Disease resistance in plants. Academic Press, New York and London. 206p.

Verma, R.P.S., Singh, D.P., Selvakumar, R., Chand, R., Singh, V.K., and Singh, A.K. 2013. Resistance to Spot Blotch in Barley Germplasm. Indian Journal of Plant Genetic Resources, 26:220225.

Wilcoxson, R.D., Rasmusson, D.C., and Miles, M.R. 1990. Development of barley resistant to leaf blight and genetics of resistance. Plant Disease, 74: 207-210.

\section{How to cite this article:}

Amrinder Kaur, Vineet K. Sharma, Simarjit Kaur, Jaspal Kaur and Chunni Lal. 2020. Characterization of Barley Entries for Spot Blotch Resistance. Int.J.Curr.Microbiol.App.Sci. 9(10): 161-171. doi: https://doi.org/10.20546/ijcmas.2020.910.021 